\title{
Evaluation of antimicrobial therapy and patient adherence in diabetic foot infections
}

\author{
Rahul Naresh Wasnik ${ }^{\mathrm{a}}$, Srujitha Marupurua ${ }^{\mathrm{a}}$, Zabiuddin Ahad Mohammed ${ }^{\mathrm{a}}$, \\ Gabriel Sunil Rodrigues ${ }^{\mathrm{b}}$, Sonal Sekhar Miraj ${ }^{\mathrm{a}, *}$ \\ a Department of Pharmacy Practice, Manipal College of Pharmaceutical Sciences, Manipal Academy of Higher Education, Manipal, India \\ ${ }^{\mathrm{b}}$ Department of Surgery, Kasturba Medical College and Hospital, Manipal Academy of Higher Education, Manipal, India
}

\section{A R T I C L E I N F O}

\section{Keywords:}

Antibiotic resistance

Diabetic foot infections

Medication adherence

Susceptibility pattern

\begin{abstract}
A B S T R A C T
Background/objectives: Diabetic foot infections (DFI) are common complications among diabetics. These wounds can cause discomfort and often become infected. In India, currently there is a paucity of data on patient adherence towards antibiotic use in DFIs. Therefore, this study was aimed to evaluate antimicrobial susceptibility pattern, antimicrobial therapy and its adherence in DFI patients.

Method: A prospective observation study ( $\mathrm{N}=150$, newly diagnosed DFI patients) was conducted in Kasturba hospital, Manipal over a period of 6 months. Culture and Sensitivity pattern of the microbes of the patients were obtained from hospital data management system to develop cumulative antimicrobial susceptibility pattern. Medication adherence was measured by using Culig adherence scale at the first follow-up visit.

Results: The most common microbes were Methicillin-sensitive Staphylococcus aureus (23.9\%), Klebsiella pneumonia (13.3\%) and Escherichia coli (13.3\%). Inj. Amoxicillin-Clavulanic acid 1.2 gm (28.6\%) was highest prescribed empirical therapy in DFI's followed by Inj. Cefuroxime-Sulbactam 2.25 gm (14.6\%), Ceftriaxone (0.7\%) and Trimethoprim- Sulfamethoxazole $(2 \%)$ was the most sensitive antibiotic. Cumulative antimicrobial susceptibility pattern shows Chloramphenicol, Colistin, Levofloxacin, Rifampicin has developed resistance towards most of the common organisms in DFI. Statistically significant association was observed between empirical therapy with T.Cefuroxime $625 \mathrm{mg}$ and improved clinical outcomes ( $\mathrm{p}<0.001$ ). Adherence shows that only $14 \%$ of patients have high adherence rates during first follow-up visit.

Conclusion: Ceftriaxone and Trimethoprim-Sulfamethoxazole are most sensitive antibiotics towards most common organisms in DFIs. T. cefuroxime $625 \mathrm{mg}$ showed statistically significant improvement in clinical outcomes while adherence is very low in most of the DFI patients.
\end{abstract}

\section{Introduction}

Diabetes mellitus (DM) is a metabolic disorder with multifactorial etiology characterized by chronic hyperglycemia with disturbances of carbohydrates, fats and protein metabolism. This results from defects in insulin secretion, insulin action or both. ${ }^{1}$ International Diabetes Federation (IDF) estimates that India ranks second after china for most diabetics. Low to middle income countries has $80 \%$ of world's diabetics. Estimated disease burden were 177 million in 2000, 285 million in $2010 .^{2}$ This value is predicted to rise to 642 million by 2040 . The number of deaths due to diabetes was estimated as 5 million until 2015. ${ }^{3}$ Globally, Diabetic foot infections (DFIs) are defined as skeletal and soft tissues infection in diabetics. In USA, incidence of amputation was 40,000 per year and incidence rate is 80,000 to one lakh amputations per year. ${ }^{4} \mathrm{DM}$ prevalence has hiked to $30 \%$ in developed countries. Furthermore, its prevalence surges with age, and more in working age population. ${ }^{5}$ It has been estimated by IDF that 193 million people, or close to half (46.5\%) of all people with diabetes, are unaware of their disease. ${ }^{6}$ In India, 69.1 million diabetics contribute the overall prevalence of $9.3 \%$. Provincial prevalence of diabetes ranges from region to region, for instance, in Jharkhand (5.3\%), Tamil Nadu (10.4\%), and Chandigarh (13.6\%). ${ }^{7}$ A community-based study from north India demonstrates that age standardized prevalence of diabetes and prediabetes were $11.2 \%$ and $13.2 \%$ respectively. It is also accounted that majority of the population remain undiagnosed $(52.1 \%)$ and this may account for another 36.1 million. ${ }^{8}$

\footnotetext{
* Corresponding author. Department of Pharmacy Practice, Manipal College of Pharmaceutical Sciences, Manipal Academy of Higher Education, Manipal, 576104, India.

E-mail address: sonal.sekhar@manipal.edu (S.S. Miraj).
} 
Diabetics with infections are difficult to treat since they have impaired micro-vascular circulation, which limits the access of phagocytic cells of infected area. This results in poor penetration of antibiotics in the infected tissues. ${ }^{9}$ Diabetics with foot infections become more severe and take longer time to cure compared to persons without diabetes. DFI occurs because of hyperglycemia and several other co-morbidities, especially neuropathy, vasculopathy, sight threatening retinopathy and defects in immunity and wound healing. DFI are the most frequent causes of hospitalization and amputation $(20 \%) .{ }^{10}$ Some studies also report that $15 \%$ of all diabetics develop foot ulcer in their lifetime. Of this around $28 \%$ of them require some kind of amputation. ${ }^{11}$ Among diabetics and non-diabetics, the risk of lower limb amputation has been accounted as 8 to 12.3 times higher. Furthermore, diabetics are 2.5 times more likely to have onychomycosis and Tinea Pedis infection that can lead to skin disruption and ulcers. In population-based studies, the annual incidence of diabetic foot ulcer, DFU is $1.0-4.1 \%$ and has a prevalence of $4.5-10 \%$, with an overall lifetime incidence of $25 \% .{ }^{12}$ All lower limbs amputations (50\%-70\%) are attributed to DFU. ${ }^{13}$ In India, a multicentric study on diabetics report prevalence of neuropathy as $15 \%$ and peripheral vascular disorder (PVD) as $5 \% .{ }^{14}$ Foot infections may results from many different microbial species (single or combination). Most of the time, response to treatment may be poor, particularly when vascular disease is also present with diabetes. Staphylococcus and Streptococci are the most commonly isolated pathogens. They were susceptible to oral antibiotic treatment like Amoxicillin-Clavulanate, Cephalexin, Clindamycin, Doxycycline, Levofloxacin, Linezolid, Minocycline, Trimethoprim-Sulfamethoxazole. For moderate to severe infections, parenteral antibiotics like Ceftriaxone, Cefoxitin, Clindamycin, Ertapenem, Daptomycin, Moxifloxacin, Piperacillin-Tazobactam, Tigecycline and Vancomycin are useful. ${ }^{15}$ Longer duration of treatment, lack of awareness and poor self-care are well known factors that culminates to medication non-adherence in DM. Patient non-adherence to therapeutic strategies is a serious concern that poses a great challenge to the successful delivery of healthcare. Prevalence of poor adherence treatment ranges from $67 \%$ to $74 \% .{ }^{16}$ Poor adherence in diabetics has been associated with increased health care resource utilization and increased rate of hospitalization. It is essential to evaluate adherence on a regular basis due to changes in culture and lifestyles, in addition to introduction of new medicines with varied dosing schedule, efficacy and adverse drug reactions, which in turn, alter adherence. In India, there are extremely limited data on systematic assessment of adherence to diabetic medication from different parts of the country. ${ }^{17}$ Hence, purpose of the study was to evaluate antimicrobial susceptibility pattern, antimicrobial therapy and its adherence in DFI patients.

\section{Methods}

This prospective observational study was carried out in Kasturba Hospital, Manipal; during the period of 6 months. All newly diagnosed patients with DFIs who were prescribed with antibiotic therapy and had an antibiotic culture and sensitivity test were included in the study. Patients excluded were of $\leq 18$ years. The detailed history of the patients, demographics, drug therapy given, and other laboratory investigations were recorded in a specially designed data collection form. To measure the adherence levels in these patients, we used the Culig adherence scale. This is a general adherence questionnaire consisting of 16 questions about the personal and emotional problems, social functioning and general health perceptions. The adherence scale provides causes of non-adherence identified by 'never', 'sometimes', 'often' and 'rarely'. Total scores represent the percentage of total possible score achieved. All items are scored with above $80 \%$ as favorable high adherence and medium score as $60-80 \%$ and low adherence as below $60 \%$.

\subsection{Study procedure}

A total of 150 diabetic foot infection patients were included for the study. The gender wise distribution of data were categorized and presented with the number of patients. The majority of the patients were male [139(92.6\%)] and whereas, female were $11(7.4 \%)$. In the adherence study among 100 patients, majority of the patients were male (91\%) and female were only $7 \%$. The susceptibility testing and sensitivity pattern were obtained from hospital lab data management system. By using this, cumulative susceptibility chart (Antibiogram) were prepared. The patients were followed from the first day of admission until discharge. Before initial antibiotic therapy, tissue or pus specimen were collected and sent for culture and susceptibility testing. Given empirical therapy, definitive therapy; the timing of empirical therapy and definitive therapy has been obtained. After discharge patients were followed at next outpatient visit at the Surgery Department. During the visit, patients were provided with self-administered Culig Adherence Scale to measure antibiotic related medication adherence levels in DFIs.

\subsection{Statistical analysis}

Nominal data were expressed as frequency and percentage. Chisquare test was performed to find association between prescribed empirical therapy and improved outcome. Data were collected and analyzed by SPSS 20.0 statistical software. A p value of $<0.05$ was considered as statistically significant.

\subsection{Ethical clearance}

Ethical clearance was obtained from the Institutional Ethical Committee of Kasturba Hospital, Manipal (Ref. No: IEC 588/2016).

\section{Results}

Among 150 DFU patients included in our study, only 100 of them completed the adherence questionnaire. Majority of the study population were males with $92.6 \%$ and $7.4 \%$ were female. Out of 150 patients, Gram-positive organisms were found in $42.5 \%$ patients, Gram-negative organisms were found in $44.6 \%$ patients and no growth of organisms was seen in $12.6 \%$ patients. Majority of Gram-positive organisms (23.9\%) were Methicillin-sensitive Staphylococcus aureus (MSSA) followed by Methicillin resistant Staphylococcus aureus (MRSA) (11.3\%) and least organism was Enterococcus faecalis (7.3\%). While in Gramnegative organisms, majority of them were Klebsiella pneumonia (13.3\%) and Escherichia coli (13.3\%) and least being Proteus mirabilis (0.7\%) and Proteus vulagris (0.7\%).

\subsection{Cumulative antimicrobial susceptibility pattern (antibiogram)}

The sensitivity patterns of causative organisms were represented in the form of antibiogram [Table 1]. Antibiotics like Chloramphenicol, Colistin, Levofloxacin, Rifampicin is found to have developed resistance for most of the organisms. Moreover, decreased sensitivity of Enterococcus faecalis, Enterococcus cloacae, Klebsiella pneumonia, MRSA and Escherichia coli towards Erythromycin, Tetracycline and Benzyl Penicillin and Ceftriaxone was also observed.

Data from 150 patients shows Inj. Amoxicillin-Clavulanic acid $1.2 \mathrm{gm}(28.6 \%)$ is the highest prescribed empirical therapy in diabetic foot Infections patients. The second highest prescribed empirical therapy is Inj. Cefuroxime -Sulbactam 2.25 gm (14.6\%). T. Cefuroxime $625 \mathrm{mg}$ (0.7\%), inj. Amikacin750 mg (0.3\%), inj. AmoxicillinClavulanic acid with inj. Cefoperazone-Sulbactam $(0.7 \%)$ was found to be least prescribed empirical therapy. The study revealed that prescribing patterns of empirical antibiotic therapy was inappropriate when compared with antibiotic policy of the hospital, 2016. 


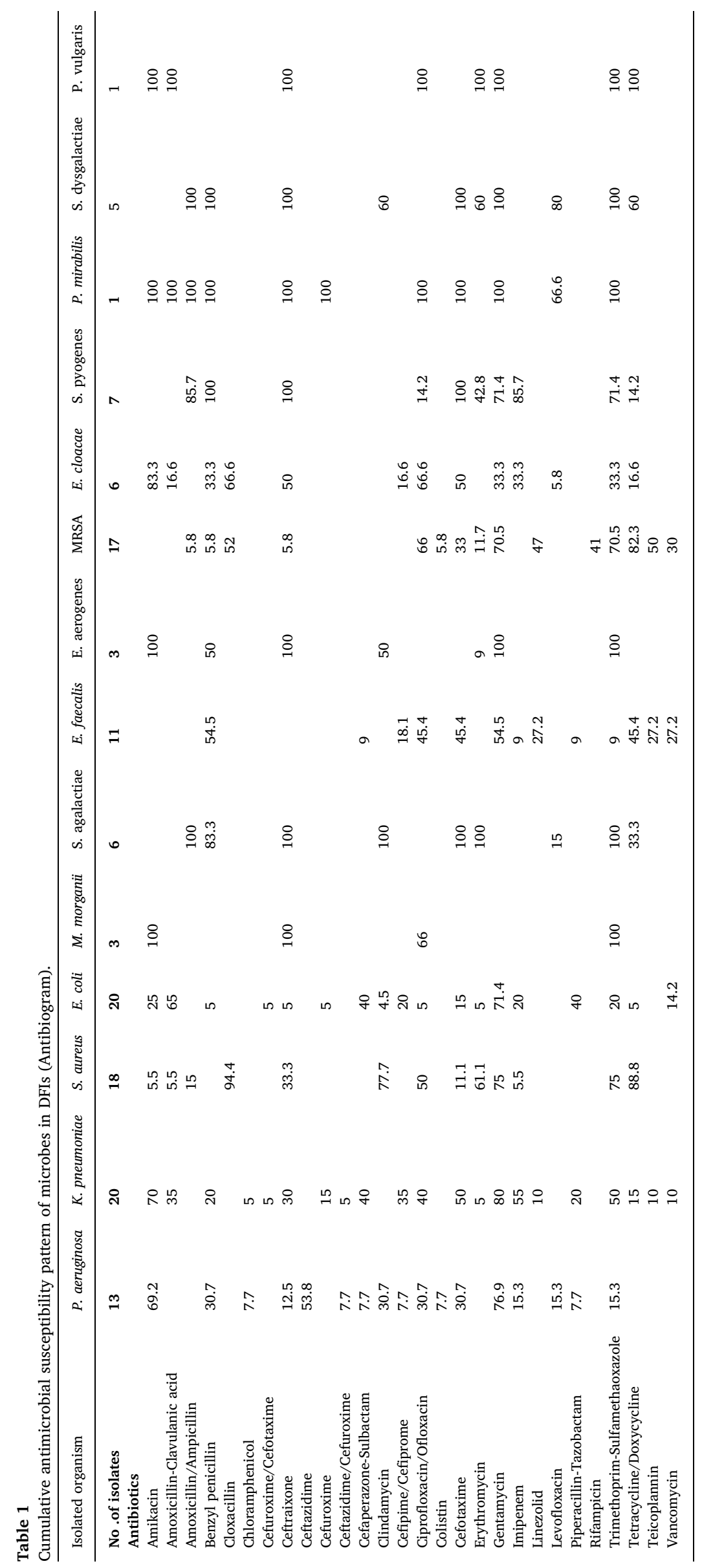


Table 2

Timeline for culture and sensitivity test after admission.

\begin{tabular}{cl}
\hline Timing & No. of patients (\%) \\
\hline$<12 \mathrm{hrs}$ & $60(40.0 \%)$ \\
$12-24 \mathrm{hrs}$ & $46(30.6 \%)$ \\
$24-48 \mathrm{hrs}$ & $21(14.0 \%)$ \\
$>48 \mathrm{hrs}$ & $6(4.0 \%)$ \\
Not done & $17(11.3 \%)$ \\
\hline
\end{tabular}

While looking at the culture and sensitivity test, in 60 (40.0\%) patients it was performed in $\leq 12 \mathrm{hrs}$ of admission and $6(4.0 \%)$ patients was performed $\geq 48 \mathrm{hrs}$ after admission [Table 2]. In $72(48.0 \%)$ patients were given empirical therapy within $\leq 12 \mathrm{hrs}$ of admission and $4(2.7 \%)$ patients were given empirical therapy $\geq 48 \mathrm{hrs}$ after admission. Only $109(72.6 \%)$ had definitive therapy given while $41(27.3 \%)$ were not given definitive therapy. We also analyzed the time taken from empirical therapy to definitive therapy after the culture and sensitivity test results were obtained. Majority of the patients, 59(54.1\%) had an average timeline of $12-24 \mathrm{~h}$ while there was just $5(4.6 \%)$ of them had therapy changed in $<12 \mathrm{~h}$.

In 65(43.3\%) patients' clinical conditions had improved and 84 $(56.0 \%)$ of patients had become stable and remaining $1(0.6 \%)$ of patient had expired. From the chi-square test, Statistically significant association was observed between T.Cefuroxime $625 \mathrm{mg}$ and improved outcomes ( $\mathrm{p}<0.001)$. While similar kind of analysis using chi-square test showed that there was no statistically significant association observed between the time of empirical therapy and improved clinical outcome ( $p=0.487)$, time of definitive therapy and improved clinical outcome $(\mathrm{p}=0.229)$ and time difference between empirical and definitive therapy $(\mathrm{p}=0.639)$.

\subsection{Measurement of antibiotic adherence}

Among 100 DFU patients, who completed the adherence questionnaire, majority of the patients were in the age group of 50-60 years ( $\mathrm{n}=33,33 \%)$, and males $91(91 \%)$. The medication adherence was assessed using Culig Adherence Scale when the patients came for first follow-up for their DFI treatment. Overall adherence patterns showed that among DFI patients there is predominantly low (44\%) and medium (42\%) adherence levels seen and only $14 \%$ of our study population showed high adherence levels of above $80 \%$.

\section{Discussion}

A total of 150 patients were included in the study for cultural sensitivity assessment. The timing of empirical therapy and definitive therapy given, and culture test timing was evaluated. In our study the Gram-positive organisms included were MSSA (23.9\%), MRSA (11.3\%), Enterococcus faecalis (7.3\%), Streptococcus pyogenes (4.6\%), Streptococcus agalactiae (4\%) and Streptococcus dysgalactiae (3.3\%). Gram-negative organisms included were Klebsiella pneumoniae (13.3\%), Escherichia coli (13.3\%), Pseudomonas aeruginosa (8.6\%), Enterobacter cloacae (4\%), Enterobacter aerogenes (2\%), Morganella morganii (2\%), Proteus mirabilis (0.7\%) and Proteus vulgaris (0.7\%).

This study concluded that all the prescribed empirical therapy for DFI is not appropriate according to the hospital policy where the study was conducted. Empiric antibiotic prescribing guidelines lead to quality and standardized care for common infectious diseases by helping prescriber's select an appropriate empirical therapy for a variety of infections. Appropriate antibiotic therapy can improve clinical outcome when administered adequately in correct dose, via the right route, given in a timely manner and with intended penetration to site of infection.

A prospective observational study conducted in surgery department of a hospital in Bengaluru, India for a period of 1 year found different results than our study and showed that culture and sensitivity test that were carried out in 17 cases and the causative organisms identified were Staphylococcus aureus, Pseudomonas species, Proteus species, Klebseilla oxytoca. The Pharmacotherapy revealed that monotherapy with antibiotics was chosen in $43.2 \%$ patients closely followed by therapy with combination of two antibiotics in $42.4 \%$ patients. Only $4 \%$ patients received more than 3 antibiotic regimens. ${ }^{18}$ In our study monotherapy with antibiotics was found to be $79.5 \%$ and dual therapy with antibiotics was found to be $3.9 \%$. A prospective study of 80 patients with DFI admitted to Cairo University hospitals found that Gramnegative bacteria accounted $56.1 \%$ and Gram-positive bacteria accounted $27.7 \%$. The common isolate was Proteus mirabilis (16.8\%) followed by Escherichia coli (13.5\%), MSSA (11.4\%), Pseudomonas species (10.8\%) and MRSA (10.1\%). Vancomycin was found to be the most effective against Gram-positive bacteria, whereas Imipenem, Amikacin and Colistin were most effective against Gram-negative bacteria. ${ }^{19}$

The timing of empirical therapy should be based on patient conditions and urgency of the situation. Initial therapy for infections can be an empirical therapy. This is a method to use wide-ranging antibiotics as initial therapy for the purpose to cover numerous pathogens commonly identifying clinical syndrome. The representation of antibiotics is to clinch the infection and ensure it does not spread across.

In majority $(40 \%)$ of the patient's culture and sensitivity test was done within $12 \mathrm{hrs}$ of their admission, 12-24hrs (30.6\%), 24-48hrs (14\%), > 48hrs (4\%) and was not done in $11.3 \%$. Time for empirical therapy within $12 \mathrm{hrs}$ was given to most of the patients (48\%), 12-24hrs (28\%), 24-48hrs (10\%), > 48hrs (2.7\%), not given in rest of the patients $(11.3 \%)$.The culture test has not been performed in $11.33 \%$ because the patient has admitted only for three days and the patients wants discharge without treatment. Definitive therapy was given to 109 $(72.6 \%)$ patients and not given in $41(27.3 \%)$ patients. Time difference between empirical therapy and definitive therapy was found to be < $12 \mathrm{hrs}$ in majority of the patients (54.1\%), 12-24hrs (36.7\%), 24-48hrs (4.6\%) and 48-72hrs (4.6\%). No growth was seen in 19 out of 150 patients. Association for empirical therapy found T. Cefuroxime $625 \mathrm{mg}$ use was statistically significant with improved clinical outcomes in DFIs ( $\mathrm{p}<0.001$ ). While, time for empirical, definitive therapy and time difference between empirical and definitive therapy was not statistically significant with improved clinical outcome.

Medication adherence is the extent to which a patient to follow the prescription prescribed by the physician to achieve better outcomes. Adhering to the medications prescribed by the physicians can lead to better health of the patient. Non-adherence is mainly due to lack of patients interest to take the medications. Method to measure adherence has been attempted in too many researches. A quality procedure must be developed to prevent complications of the problem. The absence of authentic technique for measuring non-adherence is the major obstacle in adherence research. ${ }^{19}$ Our study assessed the adherence in DFI patients and compared the appropriateness of antibiotics prescribed in these patients against the hospital antibiotic policy. We used Culig Adherence Scale for which the permission was obtained from developer, Joseph Culig as a suitable tool to evaluate medication adherence in diabetic foot ulcer (DFU) patients.

The age of the study population who participated for the medication adherence assessment were included and the maximum number of participants came under the age groups 50-60 years (33\%). Most of the studies showed that age was related to compliance. Although few researches found age being not a factor causing non-compliance. Elderly patients may have problems in vision, Hearing and memory. In addition, they may have more difficulties in following therapy instructions due to cognitive impairment or other physical difficulties, such as having problems in swallowing tablets, opening drug containers, handling small tablets, distinguishing colors or identifying marking on drugs. On the contrary, older people might also have more concern about their health than younger patients, so that older patient's nonadherence is non-intentional in most cases. ${ }^{20}$ 
Forgetfulness and multiple medications was the main reason for non-adherence in majority of our patient population while away from home and concern of side effect were less frequent reasons. Patients did not have issue with the drug whether it was expensive or not, but they just wanted to get cured as soon as possible with less financial burden and shorter duration of stay in hospital. More than three-quarter of our patients belonged to low adherence (44\%) and medium adherence (42\%). The question arises that how and why people forget to take medication. Working stress and psychosocial factors might be the reason for same. An innovative approach using modern technology for continuous reminder might help to overcome this obstacle. A Zagreb University who also did study with Culig adherence scale found that unawareness regarding the medication was the main reason for nonadherence while absence from home was the second leading reason in most age category. $66+$ age category ranked sixth to seventh place for medication non-adherence. In 56-65 age categories, away from home (52.9\%) and drug shortage were main reasons for the non-adherence $(51.2 \%) .{ }^{21}$ Male predominance was observed in our study population (91\%). This might be due to the cultural factors arising due to the decreased motivation among the females in India to seek medical consultation.

\section{Conclusion}

DFU is the major source of morbidity and mortality among diabetes patients from the past two decades. The management of DFIs is the major challenge for surgeon. This study showed most common organisms present in the DFIs were Gram-negative aerobes. However, MSSA was the most predominant organism isolated from the lesions while Inj.Amoxicillin-Clavulanic acid $1.2 \mathrm{gm}$ was most prescribed empirical therapy. The fact is that this empirical therapy was inappropriate according to hospital policy, shows importance of antibiotic treatment guidelines and strict adherence to the guidelines. Findings of this study propose that large prospective studies are essential to assess the suitable empirical antibiotic regimen in DFIs and interventions to improve glycemic control and medication adherence in DM.

\section{Sources of funding}

Nil.

\section{Conflicts of interest}

None declared.

\section{Acknowledgement}

We express our gratitude to Manipal College of Pharmaceutical Sciences and Kasturba Medical College, Manipal Academy of Higher Education, Manipal for the support.

\section{Appendix A. Supplementary data}

Supplementary data to this article can be found online at https:// doi.org/10.1016/j.cegh.2018.10.005.

\section{References}

1. Lipsky BA, Berendt AR, Cornia PB, et al. Infectious Diseases Society of America clinical practice guideline for the diagnosis and treatment of diabetic dfoot infections. Clin Infect Dis. 2012;54(12):e132-173 2012.

2. Gemechu FW, Seemant F, Curley CA. Diabetic foot infections. Am Fam Physician. 2013;88(3):177-184.

3. Morris AD, McAlpine R, Steinke D, et al. Diabetes and lower-limb amputations in the community. A retrospective cohort study. DARTS/MEMO collaboration. Diabetes audit and research in tayside scotland/medicines monitoring unit. Diabetes Care. 1998;21(5):738-743.

4. American Diabetes Association. Consensus development conference on diabetic foot wound care: 7-8 april 1999, Boston, Massachusetts. Diabetes Care. 1999;22(8):1354-1360.

5. Jeffcoate WJ. The incidence of amputation in diabetes. Acta Chir Belg. 2005;105(2):140-144.

6. Akbari CM, Macsata R, Smith BM, Sidawy AN. Overview of the diabetic foot. Semin Vasc Surg. 2003;16(1):3-11.

7. Greenman RL, Khaodhiar L, Lima C, Dinh T, Giurini JM, Veves A. Foot small muscle atrophy is present before the detection of clinical neuropathy. Diabetes Care. 2005;28(6):1425-1430.

8. Malone JM, Snyder M, Anderson G, Bernhard VM, Holloway Jr GA, Bunt TJ. Prevention of amputation by diabetic education. Am J Surg. 1989;158(6):520-523.

9. Lavery LA, Higgins KR, Lanctot DR, et al. Home monitoring of foot skin temperatures to prevent ulceration. Diabetes Care. 2004;27(11):2642-2647.

10. Gowthami B, Spurthi T, Afreen S. Drug utilization and evaluation of antibiotic in general medicine department of a tertiary care hospital. Int J Pharm Pharmaceut Sci. 2016;8(6):302-304.

11. Dinesh R, Moulya M, Abubaker S, bahrathi D. Evaluation of antibiotic usage as surgical prophylaxis in post-operative wards. Am J PharmTech Res. 2015;5:2249-3387

12. Mettler J, Simcock M, Sendi P, et al. Empirical use of antibiotics and adjustments of empirical antibiotic therapies in university hospital: a prospective observational study. BMC Infect Dis. 2007;7:21.

13. Amin N, Doupis J. Diabetic foot disease: from the evaluation of the "foot at risk" to the novel diabetic ulcer treatment modalities. World J Diabetes. 2016;7(7):153-164

14. Leese G, Nathwani D, Young M, et al. Use of antibiotics in people with diabetic foot disease: a consensus statement. Diabet Foot. 2009;12:62-78.

15. Alavi A, Bader MS, Sibbald RG. Management of diabetic foot infections with appropriate use of antimicrobial therapy. Clinical Research on Foot and Ankle. 2014 Feb 5:1-9.

16. Fincke BG, Miller DR, Christiansen CL, Turpin RS. Variation in antibiotic treatment for diabetic patients with serious foot infections: a retrospective observational study. BMC Health Serv Res. 2010 Dec;10(1):193.

17. Diabetic Foot Problems: Prevention and Management | Guidance and Guidelines. NICE; 2015. Available from: https://www.nice.org.uk/guidance/ng19.

18. Reham D, Ismail DK, Abdulbaky A. Diabetic foot infection: microbiological causes with special reference to their antibiotic resistance pattern. Egypt J Med Microbiol. 2015;24(3):95-102.

19. Morris LS, Schulz RM. Patient compliance: an overview. J Clin Pharm Therapeut. 1992;17(5):283-295.

20. Jin J, Sklar GE, Min Sen Oh V, Chuen Li S. Factors affecting therapeutic compliance: a review from the patient's perspective. Therapeut Clin Risk Manag. 2008:4(1):269-286.

21. Culig J, Leppée M, Boskovic J, Eric M. Determining the difference in medication compliance between the general patient population and patients receiving antihypertensive therapy: a case study. Arch Pharm Res. 2011;34(7):1143-1152. 